\title{
FROM DA TO SMARTGRIDS - EVOLUTION OR REVOLUTION? A PATH TO IMPROVE THE UTILITY EFFICIENCY
}

\author{
Aurélio BLANQUET \\ EDP - Portugal \\ aurelio.blanquet@edp.pt \\ Carlos TEIXEIRA \\ EDP - Portugal \\ carlosalberto.teixeira@edp.pt
}

\author{
Jorge SANTOS \\ EDP - Portugal \\ jorge.mendessantos@edp.pt \\ André MADUREIRA \\ INESC Porto - Portugal \\ agm@inescporto.pt
}

\author{
António CARRAPATOSO \\ EFACEC - Portugal \\ amc@efacec.pt
}

Fernando ALVES

noLimits Consulting - Portugal

fernando.alves@nolimits.pt

\begin{abstract}
According to the conclusions of the study "Smart Portugal 2020", the Information and Communication Technologies (ICT) can contribute to a reduction of $15 \%$ in our ecological footprint. The role of innovation and the early adoption of solutions that could reduce consumption in all economical sectors will be vital to the 20/20/20 European Strategy [1].
\end{abstract}

Active network management for generation, storage and demand is one of the basis for the SmartGrid concept, where the actions of all agents connected to the electricity system (generators, consumers and Prosumers) can be intelligently integrated aiming for a sustainable, efficient and secure energy supply system [2].

The implementation of this type of system requires an intelligent control and a management system based on advanced communication and monitoring solutions, as well as on self-healing and pre-fault detection technologies.

SmartGrids are, therefore, the most efficient approach to integrate Distributed Generation (DG) and renewable energy sources in a coordinated way with demand management in a sustainable system.

This paper describes EDP approach to the EnergyCom infrastructure, with improvement of Quality of Service $(Q o S)$ and operational efficiency as main target, and presents the importance of SmartGrids as an answer to the challenges of the Electric Energy Distribution, and its role in the corporate strategy. The 3-phase implementation program of the 3rd generation electric grid in EDP Distribuição is also presented.

\section{TELECONTROL AND AUTOMATION STRATEGY IN EDP}

Over the last 20 years [3] [4], EDP's strategy for its distribution grid was prompted by a permanent search for efficiency, focused on the Operational Expenditure (OPEX) reduction and QoS improvement, leveraged by an integrated policy of development and implementation of Substation
Automation Systems (SAS) and Medium Voltage (MV) network Distribution Automation (DA).

\begin{tabular}{|c|c|c|}
\hline Time-Scale & Main Business Drivers & ICT Strategy \\
\hline 80 's & operation cost reduction & Substation telecontrol \\
\hline 80 's - 90's & $\begin{array}{l}\text { operation cost reduction } \\
\text { Dos imnnovement }\end{array}$ & Substation automation \\
\hline 2000 & $\begin{array}{l}\text { CAPEX reduction } \\
\text { engineering cost reduction } \\
\text { maintenance cost reduction }\end{array}$ & $\begin{array}{c}\text { Standard substation design } \\
+ \\
\text { MV control project }\end{array}$ \\
\hline 2006-2007 & $\begin{array}{l}\text { Operation cost reduction } \\
\text { Further QoS improvement }\end{array}$ & $\begin{array}{c}\text { Substation design review } \\
+ \\
\text { Distribution Automation project }\end{array}$ \\
\hline 2008 - 2012 & $\begin{array}{l}\text { QoS } \\
\text { DER \& Micro generation } \\
\text { New services } \\
\text { Trans-european market } \\
\text { Ageing assets } \\
\text { Capex Constraints }\end{array}$ & Inovgrid \\
\hline
\end{tabular}

Figure 1 - EDP Telecontrol and Automation Strategy Path

In the $80 \mathrm{~s}$, the main business driver was a reduction of the operation cost, which led to a strategy of substation telecontrol to reduce OPEX. This strategy improved over the 80s and 90s, when a concern for QoS improvement led to a program of SAS, while keeping the reduction trend of OPEX by substituting the man-power by Remote Terminal Units (RTU). The number of persons that until then would control locally one substation could now control remotely 20 to 40 substations.

In the beginning of 2000, a new set of business drivers emerged: (i) Decrease the Capital Expenditure (CAPEX) by standardizing Substation design; (ii) Reduce OPEX through engineering; and (iii) Maintenance cost reduction. During these years another important program fundamental to QoS improvement was developed, the DA project, which installed more than $2000 \mathrm{MV}$ pole mounted remote controlled RTUs with simple automatisms, as well as small distribution substation (MV/LV) RTUs.

Following a 74\% improvement of the MV TIEPI (Time Interruption Equivalent Power Installed) in the last 7 years, its evolution shows a stabilization trend which evidenced the saturation of the strategy, not compatible with corporate goals demanding a continuous improvement of QoS. The increasing number of MV RTUs does not assure, by itself, a better QoS. The choice of their location in the grid must be carefully analysed to be effective. Additionally, a high number of remote controlled devices without automation 
can lead to efficiency problems on the dispatch center.

A new revolution is needed and SmartGrids can make it. To improve QoS, reduce OPEX and optimize CAPEX it is indispensable to know the real state of the grid in real time. To be more precise, it is necessary to anticipate fault situations and act before they can occur, preventing outages, planning maintenance and repairing elements during labour hours, instead of spending extra hours doing it. This is already known as $3{ }^{\text {rd }}$ Generation Electrical Grids, where one is able to integrate energy and infocommunication networks, a new concept of an EnergyCom infrastructure, applying extensively new ICT potentialities into the energy grid.

In this context, EDP launched a new Intelligent Distribution System project (InovGrid), covering from HV/MV substations to Low Voltage (LV) customers that will create a $3^{\text {rd }}$ generation technological platform.

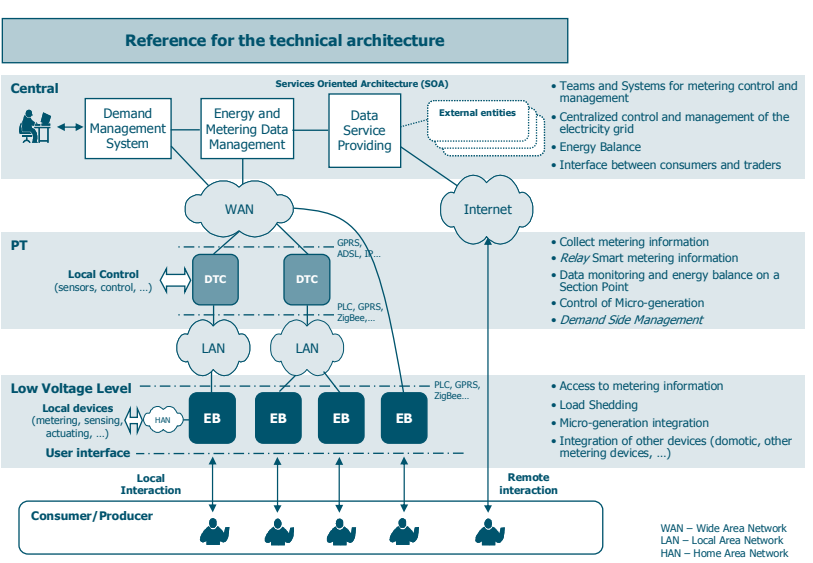

Figure 2 - InovGrid Architecture

\section{DRIVERS FOR FUTURE EVOLUTION}

The requirements of CAPEX reduction, workforce adjustments and a steady OPEX led to the need of a new vision for the sustained development of the grid, capable of facing the new sector challenges, by powering, namely, Distributed Energy Resources (DER) penetration and the development of a Pan-European energy market.

DG wide-spread can offer value to the grid system operators by providing [5] a deferral of investments to transmission and distribution systems and reduction of losses in the distribution system.

A better knowledge of both the energy flow and the grid assets' condition, as well as the possibility to control loads through Demand Response (DR), enables a new approach to network operation, supported by risk management, pre-fault detection, and dynamic reconfiguration \& self-healing capabilities, with significant impact on QoS. Better supported investment decisions and a reduction of the overall maintenance costs are also expected.

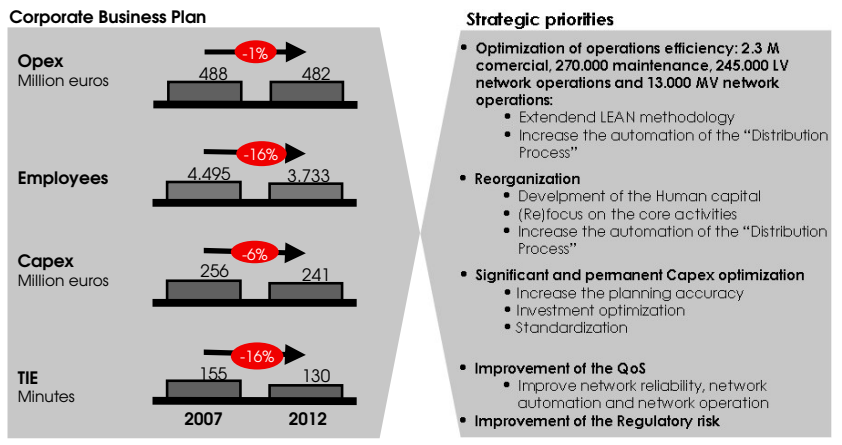

Figure 3 - Drivers for Future Evolution

\section{SMARTGRIDS AND MARKET DRIVERS}

\section{SCADA and Demand Response}

SCADA (Supervisory Control And Data Acquisition) systems are typically very "closed", with proprietary communication protocols and channels, connecting hundreds to a few thousands devices. Security is a particularly serious matter which is solved with a very controlled access, physically and virtually, to networks and devices. Although standard protocols are being implemented since the very beginning of this decade, there are still a lot of devices with proprietary communication protocols which lead commonly to a lot of time spent with conformance problems and configuration of new equipments.

To implement DR programs, it is necessary to change radically this paradigm, installing many thousands or even millions of equipments, to sensor, monitor and control each point of the network. Connect such a huge number of devices will very likely involve resorting to public communication networks. Also, extend the data network to the customer is a potential threat to the utility ICT systems by opening a back door access. Standards must be used in communication protocols and devices to avoid conformance problems between equipments. Moreover, the time needed to install and configure new equipments must not exceed a few minutes, which only will be possible by using plug \& play concepts.

ICT will be a fundamental tool to deal with large amounts of data, as well as run complex algorithms and databases, for example, to identify faults, manage grid maintenance, fraud detection, network dynamic reconfiguration, selfhealing, etc.

Presently, SCADA systems are mostly used to monitor grid faults, alarms and events and to make decisions based on current conditions. With SmartGrid and ICT integration, SCADA systems are capable of recognizing pre-fault situations and act proactively before real damages occur, using DR commands to change immediately the consumption shapes in the affected area. Additionally, 
dispatchable DER can be used to overcome power constraints.

However the big challenge in DR is the last mile. Home and Building Automation (HA and BA) have a fundamental role [6], allowing SmartGrid devices to send DR messages to a Load Management System (LMS) to control customer appliances based on pre-configured rules. Additionally, on request, LMS can inform utility systems about the available power to be reduced instantaneously, allowing some DR planning. Nowadays some HA and BA technologies, such as EIB/KNX or X10 can be used to implement DR programs; ZigBee also appears to be an important technology to be applied in classic home electric circuits.

SCADA systems and other corporate IT systems, until now independent or lightly interconnected, are moving to a strongly integrated system, enabling new algorithms to be used in the automation of the network management and operation.

\section{Microgeneration}

The need for the reduction of $\mathrm{CO}_{2}$, electricity business liberalization and technological developments have been some of the key factors responsible for the growing penetration of DG in power distribution grids.

This represents a new paradigm in electrical systems, where the generation is not purely centralized, but also relies on DER mostly based on renewable technologies such as solar and wind. Renewable energy sources will form the basis for a more sustainable energy supply system.

The large-scale deployment of these types of sources is changing power systems, moving away from centralized fuel generation systems towards environmentally friendly technologies.

The connection of generating units directly to the LV network is a reality nowadays, leading to microgrids active cells [7]. Those changes in the electrical distribution system require active network management technologies in order to efficiently accommodate large-scale integration of DG and microgeneration in coordination with DR.

\section{Commercial Systems and Demand Side Management (DSM)}

Customer service improvements will be an important benefit driven by SmartGrids. In fact, by delivering accurate and real time meter reads, the consumption estimation process will be almost eliminated for billing purposes. Providing value added services to customers, new tariffs and price plans, as well improving access to DER is bringing a new empowerment to the consumer.

Integration of Commercial and SCADA/DMS systems will allow an integrated management of DER and microgeneration, as well as DSM policies. DR systems implementation and the impacts of their peak shifting and consumption reduction will be a critical feature in the set of SmartGrid capabilities, influenced by leveraging two way communications with greater control and accuracy, and improved customer user information. This environmental benefit, driven by utilities, with the reduction of line losses and the operation of inefficient peaking power plants, is also reinforced by the continuous customer's environmental awareness leading, over time, to the replacement of inefficient equipment.

Consequently, new grid functionalities will deliver significant control to the customers in their ability to modify consumption based on the balancing of their demands and the electric system's capability to meet those demands.

The existence of a large amount of intermittent large-scale renewables on the grid infrastructure, as well as the continuous and accurate management of DG, brings to evidence the importance of commercial systems on the grid control.

As all Distibuted Energy Resources acquire a meaningful share, it will be required an accurate management of all systems involved on the distribution grid to take immediate action on a fault. New emerging products like plug-in electrical vehicles will take an active role on the electric grid (load or energy source), while also providing huge environmental benefits.

\section{EDP DISTRIBUIÇÃO STRATEGY FOR THE FUTURE}

The strategy for the future is focused on the new energy model and its alignment with the corporate strategy and business plan. The implementation of a $3^{\text {rd }}$ generation electric grid increases the ICT requirements and dependency. The number of devices on the network is increasing from several thousands to millions, and from a stable services aggregation to dynamic services integration.

This leads to the need for a trade off between cost sensitivity (huge number of devices) and complex real-time applications demanding high performance. Moreover, the installation of smart devices at the customer's premises creates the need for implementing advanced cyber-security features on the grid.

At the same time, we face the challenge of: (i) Leverage the utility strategy through the potential of the technology and in-depth knowledge of the electrical market; and (ii) Drive the technology (r)evolution and the development of intelligent distribution systems to boost the utility efficiency, increasing the automation of business processes. 
EDP identified 22 functionalities for development of the electric distribution system, ranging from assets sensing to advanced DSM and DR applications.

In this environment, complex and uncertain, EDP decided that its strategy for SmartGrids should be implemented in three phases, powering quick wins, a better team dynamics and a continuous monitoring and improvement. In order to decide the functionalities to be developed in each phase, two parameters were evaluated: (i) Impact on EDP business; and (ii) Easiness of Implementation.

The result of the evaluation is displayed in figure 4.

- 1st Phase - MV Network Sensing, Asset Management and Monitoring, Automated Service Restoration and Self-Healing, Maintenance Management

- 2nd Phase - Microgeneration Management, DER Management, Dynamic Load Forecasting, DSM and Peak Shaving, Advanced Fault Location

- 3rd Phase - Coordinated Voltage Regulation, Renewable Generation Forecasting, Advanced Service Restoration

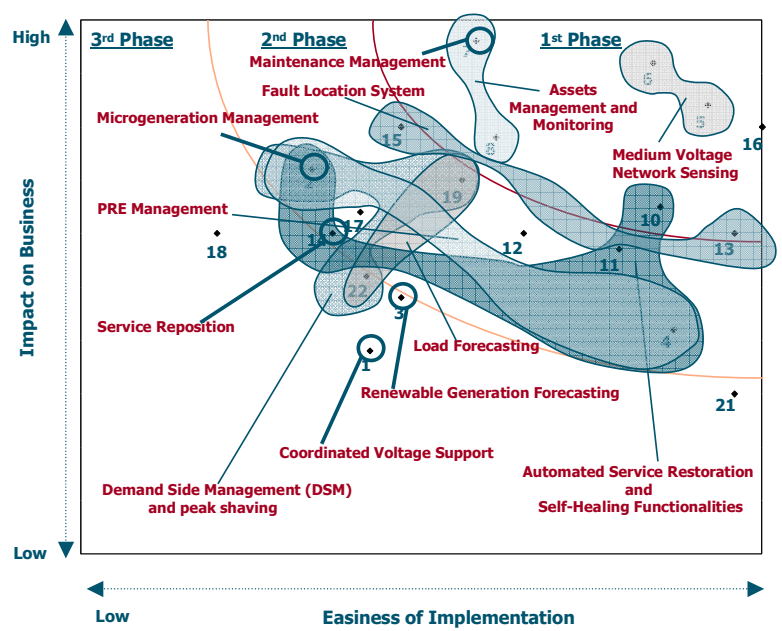

Figure 4 - Path towards the SmartGrid

\section{CONCLUSIONS}

Merging a new set of functionalities inside the existing electricity distribution grid, while keeping or excelling the current QoS level, is one of the challenges that all utilities face when deploying the SmartGrids concept.

The path toward the SmartGrid means a technological (r)evolution on the present SCADA and DA environment, made of small evolutions on devices, systems and algorithms, as well as new ones. Systems have to evolve from reactive to preventive on fault situations. The integration of DER into the electric grid and the DR program implementation will change the electric grid management, obliging a huge evolution in overall utility systems. Standards use will be very important in communications and devices.

The path defined in this paper combines a set of quick wins that minimizes the gap from present DA solutions towards the SmartGrids aproach. This roadmap is a crucial step in EDP's strategy to define grid management and control implementation of the InovGrid project. The full set of functionalities will create a $3^{\text {rd }}$ generation electric grid capable of answering the challenges of the 20/20/20 European strategy and contribute to a reduction of the Portuguese ecological footprint, combined with the EDP goals of CAPEX and OPEX reduction and QoS improvement.

\section{REFERENCES}

[1] http://eur-lex.europa.eu; 2020 by 2020 Europe's climate change opportunity.

[2] http://www.smartgrids.eu/Strategic Deployment Document for Europe's Electricity Networks of the Future, European Technology Platform - SmartGrids.

[3] João Torres; EUTC 2008 - Next Generation Utilities \& the ICT Challenge - An opportunity to leverage the electrical business efficiency.

[4] Aurelio Blanquet; European Transmission \& Distribution Summit 2008 - Boosting Quality of Service; Moving from DA Systems to Smartgrids: From reducing outage minutes to improving DSO efficiency.

[5] N. Jenkins, R. Allan, P. Crossley, D. Kirschen, G. Strbac; 2000, Embedded Generation. London, IET Power and Energy Series 31, London, United Kingdom.

[6] J. Santos, A. de Almeida, 2005, "Concepts For SCADA Systems Planning In A Changing Competitive Environment", CIRED - 18th International Conference on Electricity Distribution, Turin, Italy, Paper 352..

[7] http://www.microgrids.eu/index.php MicroGrids and More MicroGrids EU-funded projects. 